Supplement of Hydrol. Earth Syst. Sci. Discuss., 12, 7933-7969, 2015

http://www.hydrol-earth-syst-sci-discuss.net/12/7933/2015/

doi:10.5194/hessd-12-7933-2015-supplement

(C) Author(s) 2015. CC Attribution 3.0 License.

(c) (1)

Supplement of

\title{
Influence of climate variability on water partitioning and effective energy and mass transfer (EEMT) in a semi-arid critical zone
}

\section{Zapata-Rios et al.}

Correspondence to: X. Zapata-Rios (xavierzapata@email.arizona.edu)

The copyright of individual parts of the supplement might differ from the CC-BY 3.0 licence. 
Influence of climate variability on water partitioning and effective energy and mass transfer (EEMT) in a semi-arid critical zone

Supplementary Material

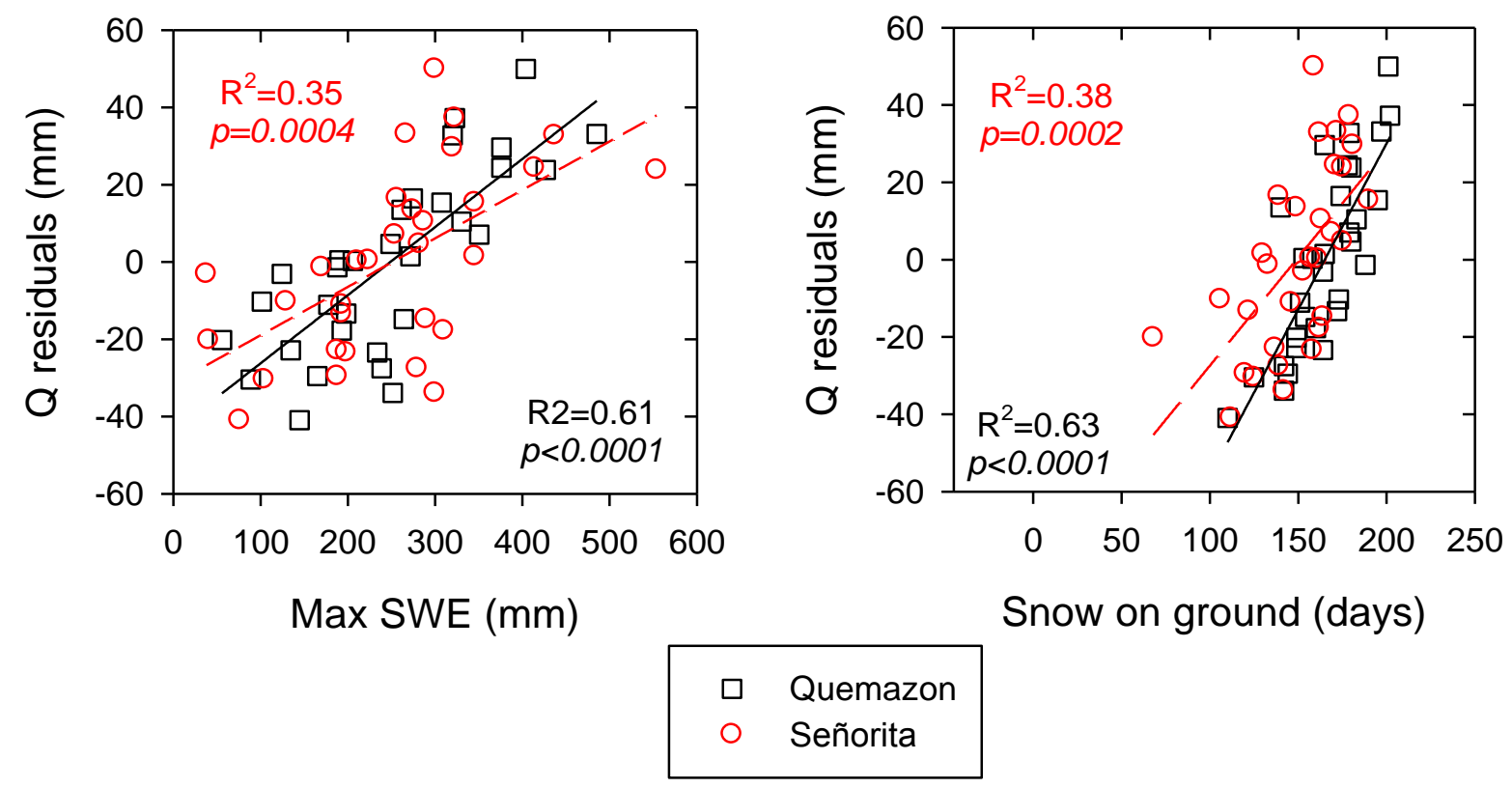

Figure S1. Plot of residuals between max SWE and snow on the ground from the linear model presented in Figure 2b. Maximum SWE and duration of the snow cover are the better predictors of discharge residuals variability. $Q$ residuals increase during extreme dry and wet years. 
Table S1. Empirical and modelled EEMT values estimated for the upper Jemez River basin.

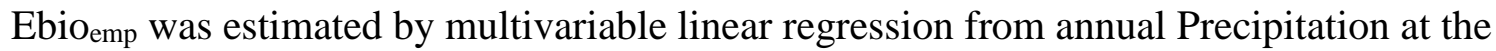
Quemazon station and Jemez River basin between 1984-1999 $\left(\mathrm{R}^{2}=0.75 ; p=0.0009\right)$

\begin{tabular}{|c|c|c|c|c|c|c|}
\hline \multirow{2}{*}{$\begin{array}{l}\text { Water } \\
\text { year }\end{array}$} & \multicolumn{3}{|c|}{$\mathrm{EEMT}_{\mathrm{emp}}$} & \multicolumn{3}{|c|}{$\mathrm{EEMT}_{\text {model }}$} \\
\hline & $\mathrm{Eppt}_{\mathrm{emp}}$ & Ebio $_{\text {emp }}$ & $\mathrm{EEMT}_{\mathrm{emp}}$ & $\mathrm{Eppt}_{\text {model }}$ & Ebio $_{\text {model }}$ & $\mathrm{EEMT}_{\text {model }}$ \\
\hline 1984 & 1.28 & 11.27 & 12.55 & 0.05 & 5.09 & 5.14 \\
\hline 1985 & 2.37 & 12.43 & 14.80 & 0.20 & 5.47 & 5.67 \\
\hline 1986 & 1.42 & 12.48 & 13.90 & 0.19 & 9.34 & 9.53 \\
\hline 1987 & 1.60 & 11.15 & 12.75 & 0.09 & 8.71 & 8.80 \\
\hline 1988 & 1.16 & 11.21 & 12.37 & 0.14 & 8.52 & 8.66 \\
\hline 1989 & 0.87 & 9.28 & 10.15 & 0.05 & 4.18 & 4.24 \\
\hline 1990 & 0.80 & 11.77 & 12.56 & 0.14 & 5.45 & 5.58 \\
\hline 1991 & 1.35 & 13.61 & 14.96 & 0.27 & 14.22 & 14.49 \\
\hline 1992 & 1.77 & 11.47 & 13.24 & 0.14 & 9.11 & 9.26 \\
\hline 1993 & 1.49 & 11.43 & 12.93 & 0.07 & 8.51 & 8.58 \\
\hline 1994 & 0.75 & 11.96 & 12.71 & 0.15 & 8.79 & 8.94 \\
\hline 1995 & 1.74 & 11.93 & 13.67 & 0.19 & 8.72 & 8.91 \\
\hline 1996 & 0.33 & 10.13 & 10.46 & 0.02 & 4.94 & 4.96 \\
\hline 1997 & 1.37 & 12.12 & 13.48 & 0.11 & 7.83 & 7.94 \\
\hline 1998 & 1.04 & 10.94 & 11.98 & 0.04 & 4.98 & 5.02 \\
\hline 1999 & 1.04 & 11.47 & 12.51 & 0.21 & 10.90 & 11.11 \\
\hline 2000 & 0.60 & 8.42 & 9.02 & 0.06 & 5.35 & 5.42 \\
\hline 2001 & 1.09 & 10.20 & 11.30 & 0.08 & 5.73 & 5.81 \\
\hline 2002 & 0.35 & 8.36 & 8.71 & 0.05 & 5.78 & 5.83 \\
\hline 2003 & 0.62 & 9.67 & 10.28 & 0.04 & 5.95 & 5.99 \\
\hline 2004 & 0.77 & 10.03 & 10.81 & 0.18 & 5.89 & 6.07 \\
\hline 2005 & 1.30 & 10.98 & 12.28 & 0.08 & 5.66 & 5.74 \\
\hline 2006 & 0.48 & 11.08 & 11.56 & 0.03 & 5.23 & 5.26 \\
\hline 2007 & 1.00 & 12.56 & 13.57 & 0.06 & 5.74 & 5.80 \\
\hline 2008 & 0.88 & 10.45 & 11.33 & 0.01 & 5.24 & 5.24 \\
\hline 2009 & 0.65 & 9.39 & 10.03 & 0.09 & 6.03 & 6.12 \\
\hline 2010 & 0.73 & 10.39 & 11.13 & 0.08 & 5.20 & 5.29 \\
\hline 2011 & 0.39 & 8.43 & 8.82 & 0.03 & 4.29 & 4.31 \\
\hline 2012 & 0.50 & 8.65 & 9.15 & 0.03 & 4.12 & 4.16 \\
\hline
\end{tabular}


Table S2. Relationship between climatic variables and discharge in the Jemez River Basin (19842012) based on records from the Quemazon and Señorita Divide\#2 SNOTEL stations. The variables are listed in decreasing order according to the linear $\mathrm{R}^{2}$ value from the Señorita Divide\#2 station.

\begin{tabular}{|c|c|c|c|c|c|c|}
\hline \multirow[b]{3}{*}{ Variable } & \multicolumn{6}{|c|}{ Discharge } \\
\hline & \multicolumn{3}{|c|}{ Quemazon station } & \multicolumn{3}{|c|}{ Señorita Divide\#2 station } \\
\hline & slope & $\mathrm{R}^{2}$ & $p$ & slope & $\mathrm{R}^{2}$ & $p$ \\
\hline Winter $\mathrm{P}(\mathrm{mm})$ & 0.18 & 0.24 & 0.00550 & 0.19 & 0.72 & 0.00001 \\
\hline 1 April SWE & 0.18 & 0.64 & 0.00010 & 0.16 & 0.56 & 0.00001 \\
\hline Max SWE (mm) & 0.23 & 0.71 & 0.00010 & 0.19 & 0.55 & 0.00001 \\
\hline last day snow cover (day) & 2.01 & 0.66 & 0.00001 & 1.83 & 0.54 & 0.00001 \\
\hline Annual P (mm) & 0.10 & 0.19 & 0.01480 & 0.13 & 0.50 & 0.00001 \\
\hline Annual temp & -11.03 & 0.27 & 0.00850 & -10.90 & 0.49 & 0.00010 \\
\hline $50 \%$ max SWE day & 1.92 & 0.48 & 0.00010 & 1.71 & 0.41 & 0.00010 \\
\hline Summer Temp & -2.04 & 0.01 & 0.58740 & -4.94 & 0.32 & 0.00320 \\
\hline Winter Temp & -8.57 & 0.17 & 0.04400 & -9.58 & 0.29 & 0.00530 \\
\hline snow in ground (days) & 0.88 & 0.44 & 0.00001 & 0.37 & 0.25 & 0.00390 \\
\hline Max SWE day & 1.15 & 0.31 & 0.00120 & 0.65 & 0.13 & 0.04800 \\
\hline 1st day snow cover (day) & -0.51 & 0.09 & 0.10290 & -0.30 & 0.12 & 0.05570 \\
\hline SWE:winter P ratio & 69.29 & 0.40 & 0.00020 & 52.28 & 0.09 & 0.09240 \\
\hline SM50 (days) & -0.22 & 0.00 & 0.73350 & 0.24 & 0.01 & 0.58760 \\
\hline
\end{tabular}


Table S3. Relationship between climatic variables and Horton index from the Jemez River basin (1984-2012) based on records from the Quemazon and Señorita Divide\#2 SNOTEL stations. The variables are listed in decreasing order according to the linear $\mathrm{R}^{2}$ value from the Señorita Divide\#2 station.

\begin{tabular}{|c|c|c|c|c|c|c|}
\hline \multirow[b]{3}{*}{ Variable } & \multicolumn{6}{|c|}{ Horton index } \\
\hline & \multicolumn{3}{|c|}{ Quemazon station } & \multicolumn{3}{|c|}{ Señorita Divide\#2 station } \\
\hline & slope & $\mathrm{R}^{2}$ & $p$ & slope & $\mathrm{R}^{2}$ & $p$ \\
\hline Winter P (mm) & -0.0001 & 0.22 & 0.00850 & -0.0001 & 0.59 & 0.00001 \\
\hline 1 April SWE & -0.0001 & 0.60 & 0.00001 & -0.0001 & 0.55 & 0.00001 \\
\hline Max SWE (mm) & -0.0002 & 0.67 & 0.00001 & -0.0002 & 0.59 & 0.00001 \\
\hline last day snow cover (day) & -0.0017 & 0.62 & 0.00001 & -0.0016 & 0.52 & 0.00001 \\
\hline Annual P (mm) & -0.0001 & 0.09 & 0.09940 & -0.0001 & 0.29 & 0.00160 \\
\hline Annual temp & 0.0103 & 0.32 & 0.00430 & 0.0086 & 0.40 & 0.00070 \\
\hline $50 \%$ max SWE day & -0.0017 & 0.50 & 0.00001 & -0.0015 & 0.41 & 0.00010 \\
\hline Summer Temp & 0.0019 & 0.01 & 0.57230 & 0.0038 & 0.25 & 0.01160 \\
\hline Winter Temp & 0.0079 & 0.19 & 0.03300 & 0.0079 & 0.26 & 0.00910 \\
\hline snow in ground (days) & -0.0007 & 0.34 & 0.00060 & -0.0004 & 0.31 & 0.00100 \\
\hline Max SWE day & -0.0010 & 0.33 & 0.00070 & -0.0006 & 0.15 & 0.03140 \\
\hline 1st day snow cover (day) & 0.0003 & 0.04 & 0.26470 & 0.0003 & 0.18 & 0.01890 \\
\hline SWE:winter P ratio & -0.0588 & 0.39 & 0.00020 & -0.0620 & 0.18 & 0.01700 \\
\hline SM50 (days) & 0.0002 & 0.01 & 0.64710 & -0.0001 & 0.00 & 0.74040 \\
\hline
\end{tabular}

